Meta

Journal des traducteurs

Translators' Journal

\title{
Hart Crane in Translation: An Updated Annotated Checklist
}

\section{Kevin J. Harty}

Volume 35, numéro 2, juin 1990

URI : https://id.erudit.org/iderudit/003037ar

DOI : https://doi.org/10.7202/003037ar

Aller au sommaire du numéro

Éditeur(s)

Les Presses de l'Université de Montréal

ISSN

0026-0452 (imprimé)

1492-1421 (numérique)

Découvrir la revue

Citer cette note

Harty, K. J. (1990). Hart Crane in Translation: An Updated Annotated Checklist. Meta, 35(2), 459-465. https://doi.org/10.7202/003037ar d'utilisation que vous pouvez consulter en ligne.

https://apropos.erudit.org/fr/usagers/politique-dutilisation/ 
HART CRANE IN TRANSLATION: AN UPDATED ANNOTATED CHECKLIST

Translations of the works of Hart Crane have previously been catalogued by Schwartz and Schweik and by Harty. This bibliographical essay updates these previous lists to include eight translations of Crane's works. These include two volumes in French, 
two in German, and one each in Italian, Japanese, Romanian, and Spanish. The staff of the Interlibrary Services Department at the National Diet Library in Tokyo generously provided me with information about and photocopies of pages from the Japanese translation, and the Biblioteca Academiei Republicii Socialiste Romania in Bucharest was kind enough to lend me a copy of the Romanian translation. As far as I have been able to determine, no library in the United States or Canada owns a copy of either the Japanese or the Romanian translations.

The primary source for listings of translations remains the new series of the international bibliography of translations, Index Translationum, published annually since 1948 in Paris by the United Nations Educational, Scientific and Cultural Organization (UNESCO).

\section{FRENCH}

BODDAERT, RICHARD, AND OTHERS, trans. (1980), Hart Crane - Poèmes et Lettres, Paris: Obisdiane.

Contains "Fear", "Interior", "At Melville's Tomb" "A Postscript", "Old Song", "Moment Fugue", "To Brooklyn Bridge", "Ave Maria", "The Harbor Dawn", "Southern Cross", "Imperator Victus", "To Liberty", "The Broken Tower", four letters, and a postcard in French and English on facing pages. Also includes a critical and biographical preface and a critical postscript in French.

GUIGUET, JEAN, ed. and trans. (1965): To Brooklyn Bridge and Other Poems/Au Pont de Brooklyn et Autres Poèmes, Paris, M. J. Minard, Lettres Modernes.

Contains "To Brooklyn Bridge", "Ave Maria", "The Harbor Dawn", "Southern Cross", "A Melville's Tomb", "Voyages II, IV, VI", and "The Broken Tower" in French and English on facing pages. Also includes an introductory biographical and critical essay in French.

\section{GERMAN}

LEISEGANG, DIETER, trans. (1968): Moment Fugue, Das Neueste Gedicht, Band 21, Darmstadt, J.G. Bläschke Verlag.

Contains "October-November", "Postscript", "Fear", "Carrier Letter", and "Moment Fugue" in German and English on facing pages. Also includes a brief chronology in German.

UHLMANN, JOACHIM, trans. (1960): Weisse Bauten, Das Neue Lot, Band 4, Berlin, Karl Henssel Verlag.

A translation of the 1926 edition of White Buildings without the foreward by Allen Tate but with a brief biographical note. German only.

\section{ITALIAN}

SANESI, ROBERTO, ed. and trans. (1967): Il Ponte e Altrie Poesie, Parma, Guanda.

Contains The Bridge and White Buildings in Italian and English on facing pages. Also includes a long critical introduction, "Elementi per Hart Crane", by Sansei and a brief critical bibliography.

\section{JAPANESE}

KURUMISAWA, ATSUO, trans. (1969): Hart Crane Shishu, Tokyo, Kokubunsha.

Contains "North Labrador", "At Melville's Tomb", "Legende", "Garden Abstract", "Repose of Rivers", "Chaplinesque", "Praise for an Urn", "Voyages I-VI", The Bridge, "O Carib Isle!", "The Idiot", "The Air Plant", "The Hurricane", and "The Broken Tower". Also includes short notes on the poems, an essay on The Bridge, a brief critical bibliography, and a short chronology. Japanese only.

\section{ROMANIAN}

MIHĂILĂ, RODICA, and IRINA GRIGORESCU, trans. (1979): Podul Brooklyn, Bucharest, Editura Univers.

Contains "Repose of Rivers", "Voyages I-VI", and The Bridge in Romanian and English on facing pages. Also includes a preface in Romanian by Mihăilă that surveys Crane's life and work.

\section{SPANISH}

BARTRA, AGUSTI trans. (1973): El Puente Ostros Poemas, Espulgas de Llobregat (Barcelona), Plaza \& Janes S.A., Editores.

Contains The Bridge, "Black Tambourine", "Emblems of Conduct", "My Grandmother's Love Letters", "Praise for an Urn", "Garden Abstract", "Pastorale", "In Shadow", "North Labrador", "Repose of Rivers", "Passage", "At Melville's Tomb", "Voyages I-VI", "Imperator Victus", "The Broken Tower", "The Invisible the Untrue", "Purgatorio", "Havana Rose", and "The Return" in Spanish and English on facing pages. Also includes an introductory bibliographical and critical essay in Spanish.

\section{WORKS CITED}

HARTY, KEVIN J. (1982): "Hart Crane in Translation: An Annotated Checklist", The Visionary Company, 1.2/2.1:181-183.

SCHWARTZ, JOSEPH, and ROBERT C. SCHWEIK 1972): Hart Crane: A Descriptive Bibliography. 
Pittsburgh: U. of Pittsburgh P - supplement in Hart Crane Newsletter 2 (1978): 47-62.

KEVIN J. HARTY

La Salle University, Philadelphia, Pennsylvania

\section{DICTIONNAIRE UNIVERSEL DES TRADUCTEURS (SUITE)}

(Voir META 32-4, 33-3, 34-4)

\section{ARCHU (XIX' s.)}

1. Traducteur français.

2. Auteur d'une traduction (1847) du plus ancien recueil de vers en langue basque du prêtre et poète Bernard Dechepare ( $\mathrm{XVI}^{\mathrm{e}} \mathrm{s}$.).

\section{ARENDT Erich (1903-)}

1. Écrivain allemand, né à Neuruppin. Exerça de nombreux métiers. Participa à la guerre civile espagnole et émigra en 1933. Après avoir beaucoup voyagé, s'est installé à Berlin-Est.

2. A traduit de l'espagnol des ceuvres des poètes latino-américains Pablo Neruda (1904-1973), Nicolas Guillén (1902-), le recueil Nackt wie der glühende Stein (1963) du Prix Nobel espagnol Vicente Aleixandre (1898 -), des poèmes de Rafael Alberti (1902-), etc.

ARENE Jules (1850- )

1. Littérateur français, né à Sisteron. Interprète de la légation de Chine à Pékin.

2. Auteur de traductions de comédies chinoises.

ARGENSOLA Bartelome (1562-1631)

1. Poète et historiographe espagnol, né à Barbastro, mort à Saragosse.

2. A traduit du latin des poésies d'Horace (65-68 av. J.-C.).

ARGHEZI Tudor (1880-1967)

1. Poète roumain, né et mort à Bucarest. De son vrai nom Ion Teodorescu.

2. A traduit du français des œuvres de Jean de La Fontaine (1621-1695), Charles Baudelaire (18211867), Arthur Rimbaud (1854-91), etc.

ARIEL M. (XIX ${ }^{\mathrm{e}} \mathrm{s}$.)

1. Orientaliste français.

2. A traduit du sanskrit la Tchaourîsouratapanchtchaschikâ (les cinquante strophes du plaisir amoureux) (1848) du poète indien Bilhana $\left(\mathrm{XI}^{\mathrm{e}} \mathrm{s}\right.$.).

ARLENIUS (XVI ${ }^{\mathbf{e}}$ s.)

1. Philosophe et littérateur flamand. Vécut en Brabant. De son vrai nom Arnould van Lens. Ami d'Henri Estienne (q.v.).

2. Traducteur d'épigrammes grecques et latines.

ARNAUD Pierre (XVII ${ }^{\mathrm{e}} \mathrm{s}$.)

1. Traductcur français.
2. A traduit le Traité de la pierre philosophale (1612) de l'alchimiste judéo-arabe Artéphius (XII ${ }^{\mathrm{e}} \mathrm{s}$.)

ARNAUD D'ANDILLY Robert (1589-1674)

1. Janséniste français, né à Paris, mort à l'abbaye de Port-Royal.

2. A traduit du grec: les Antiquités juives et la Guerre des Juifs (1681) de l'historien juif Flavius Josèphe (37-100) et collabora avec Lemaistre de Sacy (q.v.) à une traduction du Nouveau Testament (1667); du latin: les Confessions (1649) de saint Augustin (354-430), quelques livres de l'Enéide (1666) de Virgile (70-19 av. J.-C.), Du mépris du monde et La louange de l'ermite ou Eloge du désert de Lérins (1672) de saint Eucher (370-450): de l'espagnol: Quelques traités (1659) de Thérèse d'Avila (1515-82), des EEuvres (1673) de Jean de la Croix (1542-91). Ses traductions profanes illustrent l'habilité et la délicatesse avec lesquelles les traducteurs de Port-Royal modifiaient leurs originaux au nom de la morale.

\section{ARNHEIM F. (XIX' s.)}

1. Traducteur allemand

2. A traduit du finnois l'Histoire de la Finlande (1896) de Magnus Schybergson (1851-1925).

\section{ARNOLD Edwin (1832-1904)}

1. Poète et journaliste anglais, né à Gravesend, mort à Londres. Fut principal du collège sanskrit de Poona.

2. Auteur d'une traduction rimée des Cinquante strophes du plaisir amoureux et de l'Ashwagosha (1896), poèmes érotiques du brahmane Pandit Bilhana (XIe s.).

\section{ARNOLD Matthew (1822-1888)}

1. Poète et critique anglais, né à Laleham, mort à Liverpool.

2. A traduit du grec des euvres de Sophocle (497406 av. J.-C.) et d'Epictète ( $\mathrm{I}^{\mathrm{er}} \mathrm{s}$.), mais est connu surtout pour sa version de l'lliad et de l'Odyssey (1861) d'Homère (VIII' s. av. J.-C.), pleine de mesure et de subtilité, qui l'entraîna dans une polémique avec Francis Newman (q. v.), auteur d'une traduction diamétralement opposée.

A montré son intérêt pour les problèmes de la traduction par son étude On Translating Homer (1861)

\section{ARNOUX Alexandre (1884-1973)}

1. Romancier et dramaturge français, né à Digne, mort à Paris.

2. A traduit de l'espagnol La vie est un songe (1922) du dramaturge Pedro Calderón (1600-81).

\section{AROLAS Juan (1805-1849)}

1. Poète et théologien espagnol, né et mort à Barcelone.

2. A traduit du français des œuvres de Chateaubriand (1768-1848). 
AROUX E. (XIX ${ }^{\mathbf{e}}$ s.)

1. Traducteur français.

2. A traduit de l'italien La Divine Comédie (1842) de Dante (1265-1321) et, en collaboration avec P. S. Leopardi, l'Histoire universelle (19 vol., 1848-49) de l'historien Ceasare Cantú (1804-95).

ARREBO Anders (1587-1637)

1. Pasteur, évêque et poète danois, mort à Vordingborg.

2. A traduit du français la Semaine du poète protestant Guillaume du Bartas (1544-90) sous le titre Hexameron; est aussi l'auteur d'une traduction des Psaumes de David.

\section{ARRIAZA Y SUPERVIELA Juan Bautista} (1770-1837)

1. Poète espagnol, né et mort à Madrid. Diplomate en poste à Londres et à Paris.

2. A traduit du français l'Art poétique (1807) de Boileau (1636-1711).

\section{ARSENIJEVIĆ Vladan (1848-1900)}

1. Professeur yougouslave de sciences naturelles.

2. Traducteur de nombreuses œuvres littéraires russes et allemandes, notamment de la première version jouée de la tragédie Emilia Galotti (1870) de G. E. Lessing (1729-91).

ARTAUD Antonin (1896-1948)

1. Écrivain et acteur français, né à Marseille, mort à Ivry-sur-Seine.

2. A raconté en français Le Moine (1934), roman d'épouvante de Matthew G. Lewis (1775-1818).

ARTAUD M. F. (XVIII-XIX ${ }^{\mathrm{e}} \mathrm{s}$ )

1. Traducteur français.

2. A traduit de l'italien La Jérusalem délivrée (1818) du Tasse (1544-1595)

ARTAUD Nicolas (1794-1861)

1. Helléniste et littérateur français, né et mort à Paris.

2. A traduit du grec: des pièces de Sophocle (496406 av. J.-C.), Euripide (480-406 av. J.-C.) et Aristophane (445-386 av. J.-C.); du latin: des cuvres de César (101-44 av. J.-C.).

\section{ARTAUD DE MONTOR Alexis (1772-1849)}

1. Diplomate et littérateur français, né et mort à Paris. Occupa des postes en Italie.

2. A traduit de l'italien le Purgatoire (1811-13), puis la Divine Comédie (1849) complètc de Dante (1265-1321)

\section{ASAKI Georges (1788-1869)}

1. Écrivain roumain, né et mort à Jassy. Contribua puissamment à la renaissance de la littérature roumaine.

2. Également traducteur

ASCHKENASI Jakob ben Isaak (1550-1628)

1. Écrivain juif, né à Janow (Bohème), mort à Prague. Écrivait en yiddish.
2. Auteur d'une traduction-paraphrase de la Bible à l'intention des femmes, publiéc sous le titre Zeena ureena $(1590)$

\section{ASEEV Nikolaï (1889-1963)}

1. Poète futuriste russe, né à Lgov, mort à Moscou.

2. A traduit du français des poèmes de Stéphane Mallarmé (1842-98), Paul Verlaine (1844-96), Francis Viélé-Griffin (1863-1937), etc.

\section{ASÍN Y PALACIOS Miguel (XIX-XX ${ }^{\mathbf{e}}$ s.)}

1. Arabisant espagnol.

2. A traduit du mystique arabe Ibn Arabi (1165-1241) l'Épître de la sainteté dans ses Vidas de Santones Andaluces et divers passages de Révélations mecqoises dans El Islam cristianizado (1931).

\section{ASSENS Cansinos (XIX-XX ${ }^{\mathrm{e}} \mathrm{s}$ )}

1. Traducteur espagnol.

2. A traduit du russe les œuvres complètes de Dostoievski (1821-81), Tolstoï (1828-1910), Andreev (1871-1919).

\section{ASSMANN von ABSCHATZ Hans (1646-1699)}

1. Ecrivain baroque allemand, né à Würbitz, mort à Liegnitz. Études de droit à Leiden et Strasbourg. Voyages d'études à Paris, Rome et dans le Nord de l'Italie.

2. A traduit de l'italien Il Pastor Fido (1672-78), tragi-comédie pastorale du poète Battista Guarini (1370-1460), des Scherzsonnette du poète Alessandro Adimari (1579-1649), ainsi que diverses autres pièces de vers réunies sous le titre Pcetische Uebersetzungen und Gedichte (1704).

\section{AST Friedrich (1778-1841)}

1. Érudit et philosophe allemand, né à Gotha, mort à Munich. Enseigna les littératures anciennes.

2. A traduit du grec le roman d'amour Die Abenteuer von Leukippe und Kleitophon (8 vol., 1802) d'Achille Tatius ( $\mathbf{I V}^{\mathrm{e}} \mathrm{s}$.) en collaboration avec $\mathbf{G}$. Guldenapfel.

\section{ASTON W. G. (XIX-XX'}

1. Japonisant et traducteur anglais.

2. A traduit du japonais la Nihongi (Chronique du Japon, 720) sous le titre Nihongi, chronicles of Japan from the earliest times to A. D. 697 (2 vol., 1896).

\section{ASTRANA Marín (1889-1960)}

1. Critique littéraire et érudit espagnol.

2. A donné de l'anglais une version en prose des pièces de Shakespeare (1564-1616).

\section{ASTURIAS Miguel Angel (1899-1974)}

1. Écrivain et diplomate guatémaltèque, né de mère indienne à Ciudad de Guatemala, mort à Madrid. Séjours en Angleterre, en Espagne, en Grèce, en Italie, en Roumanie, en Palestine, au Mexique, au Pérou, en Argentine, en URSS, en Chine et en 
Inde. Ambassadeur du Guatémala à Paris. Prix Lénine de la Paix en 1966. Prix Nobel de littérature en 1967.

2. A traduit de l'indien quiché : le texte cosmogonique des Mayas Popol-Vuh, les Légendes des Indiens mayas (1930), etc.; du français: en collaboration avec sa femme pendant son exil à Buenos Aires (1955-62), Les Séquestrés d'Altona, Nekrassov et Le Diable et le bon Dieu de JeanPaul Sartre (1905-1980), Le Bal des toréadors de Jean Anouilh (1910), L'Herbe de Claude Simon (1913- ), Le Labyrinthe d'Alain Robbe-Grillet (1922-), etc.

\section{ATAC Nurullah (1898-1957)}

1. Essayiste et critique littéraire turc, né et mort à Istamboul. Autodidacte, acquit de vastes connaissances et une parfaite maîtrise du français au cours d'un long séjour en Suisse. Cofondateur du Bureau des Traductions (1939) qui entreprit la traduction systématique des grands chefs-d'œuvre de la littérature mondiale pour le Ministère de l'Éducation nationale. Chef du Bureau de Traduction à la présidence de la République.

2. Traducteur du français, fut le premier à faire connaître des auteurs comme Henri Michaux (1899-), etc.

\section{ATHIS Alfred (XIX-XX' $\mathbf{s}$.)}

1. Traducteur français.

2. A donné de l'anglais une adaptation de l'Admirable Crighton (1903) de l'auteur dramatique écossais James M. Barrie (1860-1937).

AUBANEL (XIX ${ }^{\mathbf{e}}$ s.)

1. Imprimeur-éditeur français.

2. A donné du grec une traduction en vers languedociens des Odes (1802) d'Anacréon (VI ${ }^{\mathrm{e}}$ s. av. J.-C.).

\section{AUBARET (XIX ${ }^{\mathfrak{e}} \mathbf{s}$.)}

1. Traducteur français.

2. A traduit de l'annamite Luc-vân-tiên (Histoire du grand lettré, 1864), le poème le plus populaire de l'Annam.

\section{AUBERTIN J. J. (XIX ${ }^{\mathrm{e}} \mathrm{s}$.)}

1. Traducteur anglais.

2. A traduit du portugais The Lusiads ( 2 vol., 1884) de Luis de Camoëns (1525-80).

\section{AUDEN Wystan Hugh (1907-1973)}

1. Poète et auteur dramatique anglais, né à York, mort à Vienne (Autriche). Émigré aux États-Unis en 1939 et naturalisé américain.

2. A traduit de 1'allemand: Songs from Mother Courage (1968) de Bertolt Brecht (1898-1956), ainsi que les livrets de The Rise and Fall of Mahagonny (1970) et Seven Deadly Sins, deux pièces du mème auteur (en collaboration avec Chester Kallman), de The magic Flute de Mozart, de Rake's Progress de Stravinski, Italienische Reise de Goethe (1749-1832), Elegy for Young Lovers de Rudolf Henz (1897-), des épigrammes du satiriste autrichien Karl Kraus (1874-1936) réunies dans The Viking Book of Aphorisms; du suédois: des poésies de Johannes Edfelt (1904-) ; etc.

\section{AUDIBERTI Jacques (1899-1966)}

1. Poète, romancier et dramaturge français, né à Antibes, mort à Paris.

2. A traduit de l'italien Les Chevaux de bois (1947), Le Chien, le photographe et le tram (1951) et d'autres cuvres du romancier Beniamino Joppolo (1906-1963)

\section{AUERBACH Berthold (1812-1882)}

1. Écrivain allemand d'origine juive, né à Nordstetten, mort à Cannes (France). De son vrai nom Moyses Baruch.

2. A traduit les cuvres (5 vol., 1841) du philosophe hollandais d'origine juive Baruch Spinoza (1632-77).

\section{AUERNHEIMER Raoul (1876-1948)}

1. Auteur et journaliste autrichien, né à Vienne, mort à Oakland (É.-U.) où il s'installa en 1938 pour fuir le nazisme.

2. A traduit du francais des œuvres de Molière (162273), Mérimée (1803-70), Paul Géraldy (1885-), etc.

\section{von AUERSPERG (cf. GRÜN Anastasius)}

\section{AUGER Athanase (1734-1792)}

1. Abbé érudit français, né et mort à Paris.

2. Auteur de traductions assez exactes mais sans couleur de classiques grecs, telles les CEuvres complètes (1774) de Démosthène (384-322 av. J.C.) et d'Eschine (390-314 av. J.C.) et les CEuvres complètes (1781) d'Isocrate (436-338 av. J.-C.), Homélies de saint Jean Chrysostome, Harangues tirées des principaux historiens grecs (1788), etc.

\section{AULARD François (1849-1928)}

1. Historien, journaliste et critique français, né à Montbron, mort à Paris. Titulaire de la chaire d'histoire de la Révolution àta Sorbonne.

2. A traduit de l'italien, avec une habilité philologique non dépourvue de dons littéraires, les Poésies et aeuvres morales (3 vol., 1880) de Giacomo Leopardi (1798-1837).

\section{AURELIANUS Caelius (IIe s.)}

1. Médecin latin.

2. A traduit du grec la Gynecia, traité chirurgical de Soranus d'Ephèse, qui vécut sous Trajan.

\section{AURELL Tage (1895-1976)}

1. Écrivain suédois, né à Oslo (Norvège), mort à Mangskog. Séjours à Berlin et en France.

2. A traduit du français: æeuvres de Stendhal (17831842); de l'allemand: des œuvres de Franz Kafka (1883-1924), Georg Büchner (1813-37), etc.; en 
français: plusieurs pièces d'August Strindberg (1849-1912).

\section{AVALICHVILI N. (XIX's $\mathbf{s}^{\mathbf{s}}$ )}

1. Traducteur géorgien.

2. A traduit de l'espagnol l'œuvre de Miguel de Cervantès (1547-1616).

\section{AVERSA Tommaso (?-1662)}

1. Poète sicilien, né à Amistrato, mort à Palerme

2. Auteur d'une traduction de l'Enéide de Virgile (70-19 av. J.-C.) en vers siciliens.

\section{AVIANUS ( $\mathbf{V}^{\mathrm{e}} \mathbf{s}$.)}

1. Fabuliste latin.

2. A traduit du grec en distiques latins, sur une version versifiée de Babrios ( $\mathrm{I}^{\mathrm{e}} \mathrm{s}$ ), deux Fables d'Esope (620-560 av. J.-C.).

\section{AVIENUS Rufus (IV's.)}

1. Poète et géographe latin, né à Volsinium.

2. A donné du grec une traduction en hexamètres des Phénomènes, poème didactique de l'astronome Aratos de Soles (315-239 av. J.-C.), ainsi que des Pronostics du même auteur.

\section{AYALA Pedro (cf. LOPEZ DE AYALA)}

von AYRENHOFF Cornelius (1733-1819)

1. Militaire et dramaturge autrichien, né et mort à Vienne. Disciple du classicisme français.

2. A traduit du français des œuvres de Boileau (16361711) et de Racine (1639-1699).

\section{AYRER Jakob (1543-1605)}

1. Dramaturge allemand, né et mort à Nuremberg.

2. Auteur d'imitations de I'anglais, notamment de The Tempest et de Much Ado About Nothing de Shakespeare (1564-1616), sous les titres respectifs de Sidea et Phänicia, ainsi que de la Spanish Trageay de Thomas Kyd (1558-94), etc.

\section{AYTOUN William (1813-1865)}

1. Écrivain et critique écossais, né à Edimbourg, mort à Blackhills / Elgin. Études de droit. Professeur de littérature anglaise.

2. Également traducteur de poésie.

\section{AZANA Y DIAZ Manuel (1880-1940)}

1. Homme d'État et littérateur espagnol, né à Alcalá de Henares, mort à Montauban (France). Études de droit en France.

2. A traduit de l'anglais, dans une langue magnifique, Bible in Spain (1921) de George Barrow.

\section{BABITS Mihály (1883-1941)}

1. Poète et traducteur hongrois, né à Szekszárd, mort à Budapest.

2. A traduit du grec: des tragédies de Sophocle (497406 av. J.-C.); du latin : Amor Sanctus, hymnes du Moyen Age; de l'anglais: le poème Le Corbeau
d'Edgar Allan Poe (1809-1849), des pièces de Shakespeare (1564-1616), des. œuvres d'Oscar Wilde (1854-1900), George Meredith (1828-1909), etc.; de l'italien: la Divina Commedia de Dante (1265-1321), deuxième traduction de cette œuvre en langue hongroise; du français : des poèmes des Fleurs du mal de Beaudelaire (1821-67) en collaboration avec Tóth (q. v.) et Kosztolányi (q. v.), des poésies de Théophile Gautier (1811-72), etc.; de l'allemand: des ouvres de Goethe (1749-1832) le Projet de paix perpétuelle d'Emmanuel Kant (1724-1804), etc.; de diverses langues: Erato, poèmes érotiques de la littérature mondiale.

\section{BABLER Otto (1901 - )}

1. Écrivain et traducteur tchécoslovaque, né à Zenica (Yougoslavie). Professeur de serbo-croate à l'université d'Olomouc. Connaissance de l'allemand, de l'anglais et du français.

2. Auteur de plus de cent-vingt traductions de diverses langues slaves, germaniques et romanes en tchèque, parmi lesquelles une version en vers de la Divina Commedia (1952) de Dante (12651321) qui connut un grand succès.

\section{BACH Rudolf (1901-1957)}

1. Dramaturge, essayiste et poète allemand, né et mort à Munich.

2. Également traducteur.

\section{BACHET DE MEZIRIAC Claude-Gaspard}

\section{(1581-1638)}

1. Mathématicien et littérateur français, né et mort à Bourg-en-Bresse. Apprit le grec, le latin, l'hébreu, l'italien et l'espagnol. Composa des vers en français, en latin et en italien. Élu à l'Académie française en 1635

2. A traduit du grec en latin: les œuvres du mathématicien Diophante (IV s.); du latin: des Epistres d'Ovide en vers français (1626), qui ne brillent guère par la fidélité. Comme théoricien, a publié un Discours sur la traduction (1635) qui s'oppose aux règles formulées un siècle plus tôt par Amyot (q. v.).

\section{BACHMANN Ingeborg (1926-1973)}

1. Femme de lettres autrichienne, née à Klagenfurt, morte à Rome.

2. A traduit de l'italien: l'cuvre du poète italien Giuseppe Ungaretti (1888-1970); de l'anglais: le drame Das Herrschaftshaus (1953) de l'écrivain américain Thomas Wolfe (1900-38).

\section{BACON Francis (1561-1626)}

1. Homme d'État, philosophe et essayiste anglais, né et mort à Londres. Précurseur de la recherche expérimentale.

2. Auteur d'un ouvrage intitule The Advancement of Learning (1605) qu'il mit lui-même en latin sous le titre De Augmentis Scientarum (1623). A traduit aussi quelques $P$ salms en anglais. 